\title{
Multiple Stellar Populations in Globular Clusters: Collection of Information from the Horizontal Branch
}

\author{
Francesca D'Antona ${ }^{1}$ and Vittoria Caloi ${ }^{2}$ \\ ${ }^{1}$ INAF - Osservatorio Astronomico di Roma, via Frascati 33, I-00040 Monte Porzio, Italy \\ email: dantona@oa-roma.inaf.it \\ ${ }^{2}$ INAF - IASF, via Fosso del Cavaliere, I-00133 Roma, Italy \\ email: vittoria.caloi@iasf-roma.inaf.it
}

\begin{abstract}
The majority of the inhomogeneities in the chemical composition of Globular Cluster (GC) stars appear due to primordial enrichment by hot-CNO cycled material processed in stars belonging to a first stellar generation. Either massive AGB envelopes subject to hot bottom burning, or the envelopes of massive fastly rotating stars could be the progenitors. In both cases, the stars showing chemical anomalies must have also enhanced helium abundance, and we have proposed that this higher helium could be at the basis of the many different morphologies of GC horizontal branches (HB) for similar ages and metallicities. The helium variations have been beautifully confirmed by the splitting of the main sequence in the clusters $\omega$ Cen and NGC 2808 , but this effect can show up only for somewhat extreme helium abundances. Therefore it is important to go on using the HB morphology to infer the number ratio of the first to the second generation in as many clusters as possible. We exemplify how it is possible to infer the presence of a $\mathrm{He}$ - rich stellar component in different clusters thanks to different HB features (gaps, RR Lyr periods and period distribution, ratio of blue to red stars, blue tails). In many clusters at least $50 \%$ of the stars belong to the second stellar generation, and in some cases we suspect that the stars might all belong to the second generation. We shortly examine the problem of the initial mass function required to achieve the observed number ratios and conclude that: 1) the initial cluster must have been much more massive than today's cluster, and 2) formation of the second stellar generation mainly in the central regions of the cluster may help in obtaining the desired values.
\end{abstract}

Keywords. globular clusters: general - globular clusters: formation - globular clusters: individual NGC 2808, NGC 6388, NGC 6441, M 3 - Stars: Horizontal Branch

\section{Introduction}

The observations of GC stars are still to be interpreted in a fully consistent frame. Nevertheless, a general consensus is emerging on the fact that most GCs can not be considered any longer "simple stellar populations", and that "self - enrichment" is a common feature among GCs. This consensus has been the consequence of three independent lines of evidence:

- Spectroscopic observations: the discovery of "chemical anomalies", such as the Na - $\mathrm{O}$ and $\mathrm{Mg}-\mathrm{Al}$ anticorrelation, dates back to the seventies. The anomalies are now observed also at the turnoff (TO) and among the subgiants (e.g.,Gratton et al. 2001, Briley et al. 2002, 2004), so they must be attributed to some process of "self - enrichment" occurring at the first stages of the cluster life. There must have been a first epoch of star formation that gave origin to the "normal" (first generation) stars, with CNO and other abundances similar to the population II field stars of the same metallicity. Afterwards, there must have been some other epoch of star formation, including material heavily 
processed through the CNO cycle. This material either was entirely ejected by stars belonging to the first stellar generation, or it is a mixture of ejected and pristine matter of the initial star forming cloud. We can derive this conclusion as a consequence of the fact that there is no appreciable difference in the metallicity of the "normal" and chemically anomalous stars belonging to the same GC. (Needless to say, this statement does not hold for $\omega$ Cen, which must indeed be considered a small galaxy and not a typical GC. In the following, we will only examine "normal clusters", those which do not show signs of metal enrichment due to supernovae ejecta). This is an important fact that tells us, e.g., that it is highly improbable that the chemical anomalies are due to mixing of stars born in two different clouds, as there is no reason why the two clouds should have a unique metallicity. In addition, the clusters showing chemical anomalies have a huge variety of metallicities, making the suggestion of mixing of two different clouds even more improbable. The matter must have been processed through the hot CNO cycle, and not, or only marginally, through the helium burning phases, as the sum of CNO elements is the same in the "normal" and anomalous stars (Cohen \& Meléndez 2005). The progenitors then may be either massive Asymptotic Giant Branch (AGB) star (Ventura et al. 2001, 2002) or fast rotating massive stars (Decressin et al. 2007).

- Interpretation of the Horizontal Branch morphology in terms of helium content variations among the GC stars. Whichever the progenitors, some helium enrichment must be present in the matter processed through hot CNO. D'Antona et al. (2002) recognized that this could have a strong effect on the Horizontal Branch (HB) morphology, and even help to explain some features (gaps, hot blue tails, second parameter effect) which had defied all reasonable alternative explanation. A wide variety of problems has been examined in the latest years: the very peculiar morphology of the HB in the massive cluster NGC 2808, interpreted in terms of varying helium among its stars; the second parameter effect in the clusters M 13 and M 3 (Caloi \& D'Antona, 2005); the very peculiar features of the GCs NGC 6441 and NGC 6388 (Caloi \& D'Antona, 2007a), which can all be modeled by assuming that quite a large fraction of stars have high helium. Hints that this was correct came from the analysis of the helium content in hot HB stars (Moehler et al. 2004).

- Photometric splitting of the main sequence in a few clusters. The necessity of a varying helium content in NGC 2808 was later confirmed by the first analysis of the cluster main sequence (D'Antona et al. 2005), that showed a tail of "blue" stars. This could only be interpreted as a very helium rich sequence. In fact, Carretta et al. (2006) had shown that the metallicity of oxygen poor and oxygen normal stars is the same. The recent new HST observations by Piotto et al. (2007) leave no doubt that there are at least three different populations in this cluster. This came after the first discovery of a peculiar blue main sequence in $\omega$ Cen (Bedin et al. 2004), interpreted again in terms of a very high helium content (Norris 2004, Piotto et al. 2005). The HB observations however have shown that the blue main sequences are only the tip of the iceberg of the self - enrichment. In most clusters the higher helium abundances remain confined below $\mathrm{Y} \sim 0.30$, and the presence of such stars will not be clearcut from main sequence observations (D'Antona et al. 2002, Salaris et al. 2006). Viceversa, if we wish to shed light on the entire process of formation of GCs we must have a rough idea of the total number of chemically anomalous stars.

Consequently, we decided to continue our investigation of the HB in as many clusters as possible, in the hypothesis that the HB morphology can be mainly interpreted in terms of a helium content distribution among the stars. We summarize here some of the results, as a basis to discuss the initial mass function of the first generation, required to produce the needed fraction of stars in the second generation. 


\section{Different clusters, different necessity for helium rich stars}

We recall that the role of helium rich stars is different according to which is the basic HB morphology of the first generation stars (D'Antona et al. 2002).

\subsection{NGC 2808}

If the age, metallicity and mass loss are such that normal - helium stars populate a red clump, the stars with helium enhancement (which are less massive) will populate the bluer HB and the RR Lyr region. If there is a gap between the normal - helium stars and the minimum helium content of the second generation (a situation which does probably occur, if the processed matter comes from the massive AGBs), the case of NGC 2808 shows up: a red clump (first generation), almost no RR Lyr (due to the helium gap) and a blue part with larger helium content ( $\mathrm{Y} \sim 0.28$ according to D'Antona and Caloi 2004). In addition, the presence of a separate very high helium population -as derived from the main sequence- may explain the two blue tails of the HB (D'Antona et al. 2005). The cluster seems to be divided into 50\% normal - helium stars, and 50\% helium enriched stars, but remember that the very high helium $(\mathrm{Y} \sim 0.40)$ stars are only $\sim 15 \%$.

\subsection{NGC 6441 and NGC 6388. And also 47 Tuc}

The case of these two high metallicity clusters is even more interesting: here the red clump extends for about a magnitude thickness, and any attempt to attribute this to differential reddening has failed (Raimondo et al. 2002). The RR Lyr have a very long period, unexplicable for the metallicity (Pritzl et al. 2000). And the HB extends even to the region of blue, hot stars. Caloi \& D'Antona(2007a) show that this is another case study: the morphology requires not only some helium enrichment for the bluer side of the $\mathrm{HB}$, as we could naively think, but extreme helium enrichment even for the red clump stars! In fact, the high helium - high metallicity helium core burning low mass stars make long loops from red to blue in the HB (Sweigart \& Gross 1976). This is due to the fact that the higher mean molecular weight - leading to a high $\mathrm{H}$ - burning shell temperature - and the high metallicity - leading to a stronger CNO shell - both conspire towards the result that the $\mathrm{H}$ - shell energy source prevails with respect to the $\mathrm{He}-$ core burning. The consequent growth of the helium core leads evolution towards the blue. Therefore, if we must explain the luminous (long period) RR Lyr by stars having high helium, the same stars will also populate the red clump: this is exactly what we observe: if the helium content is not as large as $\mathrm{Y} \sim 0.35$ in the red clump, the HB finds no satisfactory explanation. The percentage of helium enriched stars is in this case $\sim 60 \%$ for NGC 6441 and the same for NGC 6388. The main difference among the two clusters is that NGC 6388 seems to have a higher tail of very high helium (Y>0.35) stars, reaching $\sim 20 \%$. If we analyze 47 Tuc, the prototype of metallic GCs, the red clump seems to imply higher helium only for $\sim 25 \%$ of the stars. Why a cluster almost as massive as the other two is so different - more normal - remains to be explained.

\subsection{M3 and the problem of RR Lyr}

The case of M3 is entirely different: it has a well populated red HB, variable region and blue side ( $\mathrm{R}, \mathrm{V}$ and $\mathrm{B}$ samples), with no blue tails, so it has always been taken as the prototype of HBs: its color distribution can be reproduced by assuming an average mass loss along the RGB, with a standard deviation $\sigma \sim 0.025 \mathrm{M}_{\odot}$. Unfortunately, the RR Lyr period distribution is not so easily explained, as it is terribly peaked! Castellani et al. (2005) realized that the only way to reproduce this peak was to reduce the dispersion in mass loss rate: unfortunately, they also had to add a different average mass loss, with a different spread, to account for the blue side of the HB! Of course, if we assume that the 
Table 1. Helium history of 8 clusters

\begin{tabular}{|c|c|c|c|c|c|c|c|c|c|c|c|c|c|c|c|}
\hline \multicolumn{2}{|c|}{ NGC 2808} & \multicolumn{2}{|c|}{ NGC 6441} & \multicolumn{2}{|c|}{ NGC 6388} & \multicolumn{2}{|c|}{47 Tuc } & \multicolumn{2}{|c|}{ M3 } & \multicolumn{2}{|c|}{ M 5} & \multicolumn{2}{|c|}{ M53 } & \multicolumn{2}{|c|}{ M13 } \\
\hline $\mathrm{Y}$ & $\%$ & Y & $\%$ & $\mathrm{Y}$ & $\%$ & $\mathrm{Y}$ & $\%$ & $\mathrm{Y}$ & $\%$ & $\mathrm{Y}$ & $\%$ & $\mathrm{Y}$ & $\%$ & $\mathrm{Y}$ & $\%$ \\
\hline 0.24 & 50 & 0.25 & 38 & .25 & 39 & .25 & 75 & .24 & .50 & .24 & . 40 & \begin{tabular}{|l}
.24 \\
.
\end{tabular} & 1. & .24 & 0.0 \\
\hline $0.26-0.29$ & 35 & $0.27-0.35$ & 48 & $0.27-0.35$ & 41 & $0.27-.32$ & 25 & .26 & 50 & .26 & 60 & $>.24$ & 0. & .28 & 1.0 \\
\hline$\sim 0.4$ & 15 & $>0.35$ & 14 & $>0.35$ & 20 & & & & & & & & & & \\
\hline
\end{tabular}

blue side is populated by helium rich stars, the conundrum is solved, as shown by Caloi \& D'Antona, 2007b. In our simulations, we can explain both the period distribution and the color distribution along the $\mathrm{HB}$ for the $\mathrm{R}, \mathrm{V}$ and $\mathrm{B}$ regions. This analysis poses another problem: we find that the dispersion in mass loss along the red giant branch must be at most $\sigma \sim 0.003 \mathrm{M}_{\odot}$ to be consistent with the period distribution. We now pose the question whether this small dispersion is peculiar to M3 or we have always been mislead by the HB morphology, when assuming dispersion in mass loss of some hundreths of $\mathrm{M}_{\odot}$.

\subsection{Preliminary analysis of a few other clusters}

We list in Table 1 the results of the synthetic HB computations, including also the preliminary analysis for some other clusters. M5 is similar to M3, but has a larger fraction of higher helium stars (the peak of the number of stars is in the blue HB). M53 is a massive cluster, but its entirely blue HB could be explained by a single (normal) stellar population, maybe with a small tail of stars having slightly larger mass loss. We list in the table also M13, for which the analysis implying that only the second generation has survived in the cluster is taken from the relative location of the red giant bump, the turnoff and the HB (Caloi \& D'Antona, 2005).

\section{How did the GCs form?}

Any model for the GC formation must be able to deal with this variety of results: there are clusters with no self - enrichment, and clusters which might have lost entirely their first generation. It is true that the most massive clusters have extreme helium enhancements, but also moderately massive clusters show considerable degrees of helium variations, and a small cluster like NGC 6397, which is apparently monoparametric, might be composed entirely of second generation stars: in fact the nitrogen abundance of all its stars is severely enhances, like in CNO processed material.

The typical situation is than $50 \%$ normal - helium stars and $50 \%$ enhanced - helium stars. It is almost obvious that the ejecta of a unique first stellar generation with a normal initial mass function (IMF) can not produce enough mass to give origin to such a large fraction of second generation stars (see, e.g., the case made by Bekki and Norris (2004), for the blue main sequence of $\omega$ Cen).

The only solution to this problem is that the starting initial mass from which the first generation is born was MUCH LARGER than todays first generation remnant mass (a factor 10 to 20 larger), so that the processed ejecta of the first generation provide enough mass to build up the second one. It is possible that a cooling flow collects the gas in the core, and that the second generation stars are preferentially born there (D'Ercole et al. , in preparation). This formation in the central cluster region may also by helped if the massive (or intermediate mass stars) progenitors were already segregated in the core when the second generation is born, as suggested by the observations of young clusters. 
When the long term evolution leads to the loss of the external parts of clusters, these are populated preferentially by the first generation stars and the desired ratio of first to second generation can be achieved.

In some sense, the study of chemical anomalies leads us to the idea that practically the $G C$ forms in the second stellar generation, and the remnant first generation is only the core fraction of the much less concentrated and wider system whose winds, collected in a cooling flow, gave birth to the peculiar stars! Should we then expect different velocity dispersions in first and second generation? Would the second generation at least in some cases- remain more concentrated than the first one? of course complete dynamical simulations of the fluid gas formation phase, coupled with $\mathrm{N}$ - body simulations to describe the interaction of the two stellar generations are needed to answer this question.

\section{Acknowledgements}

We would like to thank P. Ventura, A. D'Ercole and E. Vesperini for their support and collaboration through this adventure of building up a new view of globular clusters. We also thank the organizers of the IAU Symposium 246 for their successful effort to prepare a scientific and environmentally attractive meeting.

\section{References}

Bedin, L. R., Piotto, G., Anderson, J., Cassisi, S., King, I. R., Momany, Y., Carraro, G. 2004, ApJ, 605, L125

Bekki, K. \& Norris, J. E. 2006, ApJ Letters, 637, L109

Briley, M. M., Cohen, J. G., \& Stetson, P. B. 2002, ApJ Letters, 579, L17

Briley, M. M., Harbeck, D., Smith, G. H., \& Grebel, E. K. 2004, AJ, 127, 1588

Caloi, V. \& D’Antona, F. 2005, A\&A A, 121, 95

Caloi, V. \& D'Antona, F. 2007, A\&A, 463, 949

Caloi, V. \& D'Antona, F. 2007, ApJ, in press

Carretta, E., Bragaglia, A., Gratton, R. G., Leone, F., Recio-Blanco, A., \& Lucatello, S. 2006, $A \& A, 450,523$

Castellani, M., Castellani, V., \& Cassisi, S. 2005, A\&A, 437, 1017

Cohen, J. G. \& Meléndez, J. 2005, AJ, 129, 303

D’Antona, F., Caloi, V., Montalbán, J., Ventura, P., \& Gratton, R. 2002, A\&\&A 395, 69

D'Antona, F. \& Caloi, V. 2004, ApJ, 611, 871

D'Antona, F., Bellazzini, M., Caloi, V., Fusi Pecci, F., Galleti, S., \& Rood, R. T. 2005, ApJ, 631,868

Decressin, T., Meynet, G., Charbonnel, C., Prantzos, N., \& Ekström, S. 2007, A $\varepsilon A, 464,1029$

Gratton, R. G. et al. 2001, A\&A, 369, 87

Moehler S., Sweigart A.V., Landsman W.B., Hammer N.J., \& Dreizler S. 2004, A\&A, 415, 313

Norris, J. E. 2004, ApJ Letters, 612, L25

Piotto, G., et al. 2005, ApJ, 621, 777

Piotto, G., et al. 2007, ApJ Letters, 661, L53

Pritzl, B., Smith, H. A., Catelan, M., \& Sweigart, A. V. 2000, ApJ Letters, 530, L41

Raimondo, G., Castellani, V., Cassisi, S., Brocato, E., \& Piotto, G. 2002, ApJ, 569, 975

Salaris, M., Weiss, A., Ferguson, J. W., \& Fusilier, D. J. 2006, ApJ, 645, 1131

Sweigart, A. V., \& Gross, P. G. 1976, ApJ Suppl. Series, 32, 367

Ventura, P., D'Antona, F., Mazzitelli, I., \& Gratton, R. 2001, ApJ Letters, 550, L65

Ventura, P., D’Antona, F., \& Mazzitelli, I. 2002, A\&A, 393, 215 